\title{
Characterisations of elementary pseudo-caps and good eggs
}

\author{
Sara Rottey \\ Department of Mathematics \\ Vrije Universiteit Brussel \\ Pleinlaan 2, 1050 Brussel, Belgium \\ srottey@vub.ac.be
}

\author{
Geertrui Van de Voorde* \\ Department of Mathematics \\ Universiteit Gent \\ Krijgslaan 281, S22, 9000 Gent, Belgium \\ gvdvoorde@cage. ugent. be
}

Submitted: Dec 16, 2014; Accepted: Feb 5, 2015; Published: Feb 25, 2015

Mathematics Subject Classifications: 51E20, 05B25

\begin{abstract}
In this note, we use the theory of Desarguesian spreads to investigate good eggs. Thas showed that an egg in $\mathrm{PG}(4 n-1, q), q$ odd, with two good elements is elementary. By a short combinatorial argument, we show that a similar statement holds for large pseudo-caps, in odd and even characteristic. As a corollary, this improves and extends the result of Thas, Thas and Van Maldeghem (2006) where one needs at least 4 good elements of an egg in even characteristic to obtain the same conclusion. We rephrase this corollary to obtain a characterisation of the generalised quadrangle $T_{3}(\mathcal{O})$ of Tits.

Lavrauw (2005) characterises elementary eggs in odd characteristic as those good eggs containing a space that contains at least 5 elements of the egg, but not the good element. We provide an adaptation of this characterisation for weak eggs in odd and even characteristic. As a corollary, we obtain a direct geometric proof for the theorem of Lavrauw.
\end{abstract}

Keywords: Pseudo-caps; Eggs; Translation generalised quadrangles

\section{Preliminaries}

In this note, we study eggs and pseudo-caps in the projective space $\mathrm{PG}(n, q)$, where $\operatorname{PG}(n, q)$ denotes the $n$-dimensional projective space over the finite field $\mathbb{F}_{q}$ with $q$ elements, $q=p^{h}, p$ prime. Many previous proofs and characterisations of eggs rely on the connection with eggs and translation generalised quadrangles [16]. It is our aim to study eggs from a purely geometric perspective, without using this connection or coordinates.

*Supported by the Fund for Scientific Research Flanders (FWO - Vlaanderen). 
In Section 2 we obtain a connection between good eggs and Desarguesian spreads. This link will enable us to reprove, improve or extend known results in Sections 3 and 4 . We begin by repeating some well-known definitions.

Definition 1. A cap in $\operatorname{PG}(n, q)$ is a set of points such that every three points span a plane. A cap of size $k$ is denoted as a $k$-cap.

A $k$-cap of $\mathrm{PG}(2, q)$ is often called a $k$-arc. A $k$-arc in $\mathrm{PG}(2, q)$ satisfies $k \leqslant q+1$ for $q$ odd and $k \leqslant q+2$ for $q$ even. A $(q+1)$-arc is called an oval, a $(q+2)$-arc a hyperoval. A $k$-cap of $\mathrm{PG}(3, q), q>2$ satisfies $k \leqslant q^{2}+1$, moreover, a $\left(q^{2}+1\right)$-cap of $\mathrm{PG}(3, q)$ is often called an ovoid. We will consider the higher dimensional equivalent of these structures.

Definition 2. A pseudo-cap is a set $\mathcal{C}$ of $(n-1)$-spaces in $\operatorname{PG}(2 n+m-1, q)$ such that any three elements of $\mathcal{C}$ span a $(3 n-1)$-space.

If $m=n$, a pseudo-cap is often called a pseudo-arc. By [15], a pseudo-arc $\mathcal{A}$ in $\operatorname{PG}(3 n-1, q)$ satisfies $|\mathcal{A}| \leqslant q^{n}+1$ for $q$ odd and $|\mathcal{A}| \leqslant q^{n}+2$ for $q$ even. If a pseudo-arc $\mathcal{A}$ has $q^{n}+1$ or $q^{n}+2$ elements, $\mathcal{A}$ is a pseudo-oval or pseudo-hyperoval respectively. If $m=2 n$, a pseudo-cap with $q^{2 n}+1$ elements is called a pseudo-ovoid.

Examples of pseudo-caps in $\mathrm{PG}(k n-1, q)$ arise by applying field reduction to caps in $\mathrm{PG}\left(k-1, q^{n}\right)$ and if a pseudo-cap is obtained by field reduction, we call it elementary. Field reduction is the concept where a point in $\mathrm{PG}\left(k-1, q^{n}\right)$ corresponds in a natural way to an $(n-1)$-space of $\mathrm{PG}(k n-1, q)$. The set of all points of $\mathrm{PG}\left(k-1, q^{n}\right)$ then correspond to a set of disjoint $(n-1)$-spaces partitioning PG $(k n-1, q)$, forming a Desarguesian spread. Every Desarguesian spread $\mathcal{D}$ has the property that the space spanned by 2 elements of $\mathcal{D}$ is partitioned by elements of $\mathcal{D}$, i.e. $\mathcal{D}$ is normal. Moreover, a normal $(n-1)$-spread of $\mathrm{PG}(k n-1, q), k>2$, is Desarguesian, see [2]. For more information on field reduction and Desarguesian spreads we refer to [9].

A partial spread in $\mathrm{PG}(n+m-1, q)$ is a set of mutually disjoint $(n-1)$-spaces. Every element $E_{i}$ of a pseudo-cap $\mathcal{E}$ of $\mathrm{PG}(2 n+m-1, q)$ defines a partial spread

$$
\mathcal{S}_{i}:=\left\{E_{0}, \ldots, E_{i-1}, E_{i+1}, \ldots, E_{|\mathcal{E}|}\right\} / E_{i}
$$

in $\mathrm{PG}(n+m-1, q) \cong \mathrm{PG}(2 n+m-1, q) / E_{i}$ and we say that the element $E_{i}$ induces the partial spread $\mathcal{S}_{i}$.

A partial spread of $\operatorname{PG}(2 n-1, q)$ of size $q^{n}$ is said to have deficiency 1. From [3], we know that a partial spread of $\operatorname{PG}(2 n-1, q)$ with deficiency 1 can be extended to a spread in a unique way, i.e. the set of points in $\operatorname{PG}(2 n-1, q)$ not lying on an element of the partial spread, form an $(n-1)$-space.

Definition 3. A weak egg in $\mathrm{PG}(2 n+m-1, q)$ is a pseudo-cap of size $q^{m}+1$.

Clearly, pseudo-ovals and pseudo-ovoids are examples of weak eggs. A weak egg $\mathcal{E}$ in $\mathrm{PG}(2 n+m-1, q)$ is called an egg if each element $E \in \mathcal{E}$ is contained in a $(n+m-1)$-space, $T_{E}$, which is skew from every element of $\mathcal{E}$ different from $E$. The space $T_{E}$ is called the tangent space of $\mathcal{E}$ at $E$. It is not hard to show that if $n=m$, then every weak egg is an 
egg. Eggs are studied mostly because of their one-to-one correspondance with translation generalised quadrangles of order $\left(q^{n}, q^{2 n}\right)$, see Subsection 3.2.

The only known examples of eggs in $\mathrm{PG}(2 n+m-1, q)$ have either $m=n$ or $m=2 n$ and we have the following theorem restricting the number of possibilities for the parameters $n$ and $m$.

Theorem 4. [11, Theorem 8.7.2] If $\mathcal{E}$ is an egg of $\mathrm{PG}(2 n+m-1, q)$ then $m=n$ or $m a=n(a+1)$ with a odd. Moreover, if $q$ even, then $m=n$ or $m=2 n$.

This explains why the study of eggs is mainly focused on pseudo-ovals and pseudoovoids.

In the case of pseudo-ovals, all known examples are elementary. The classical example of an oval in $\mathrm{PG}\left(2, q^{n}\right)$ is a conic. It is a well-known theorem of Segre that an oval of $\mathrm{PG}\left(2, q^{n}\right), q$ odd, is always a conic. A pseudo-conic in $\mathrm{PG}(3 n-1, q)$ is an elementary pseudo-oval, arising from applying field reduction to a conic in $\operatorname{PG}\left(2, q^{n}\right)$. We have the following theorems characterising elementary pseudo-ovals using the induced Desarguesian spreads.

Theorem 5. [6] If $\mathcal{O}$ is a pseudo-oval in $\mathrm{PG}(3 n-1, q)$, $q$ odd, such that for at least one element the induced spread is Desarguesian, then $\mathcal{O}$ is a pseudo-conic.

Theorem 6. [13] If $\mathcal{O}$ is a pseudo-oval in $\mathrm{PG}(3 n-1, q)$, $n$ prime, $q>2$ even, such that all elements induce a Desarguesian spread, then $\mathcal{O}$ is elementary.

In the case that $q$ is odd, we have the following theorem which extends Theorem 5 from pseudo-ovals to large pseudo-arcs in $\mathrm{PG}(3 n-1, q)$.

Theorem 7. [12] Consider $\mathcal{K}=\left\{K_{1}, \ldots, K_{s}\right\}$ a pseudo-arc in $\mathrm{PG}(3 n-1, q)$, q odd, of size greater than the size of the second largest complete arc in $\mathrm{PG}\left(2, q^{n}\right)$. If for at least one element $K_{i}$ of $\mathcal{K}$, the partial spread $\mathcal{S}=\left\{K_{1}, \ldots, K_{i-1}, K_{i+1}, \ldots, K_{s}\right\} / K_{i}$ extends to a Desarguesian spread of $\mathrm{PG}(2 n-1, q)=\mathrm{PG}(3 n-1, q) / K_{i}$, then $\mathcal{K}$ is contained in a pseudo-conic.

In Theorem 15, we will prove a similar statement for pseudo-caps in $\mathrm{PG}(4 n-1, q)$.

All known examples of pseudo-ovoids in $\mathrm{PG}(4 n-1, q)$ are elementary when $q$ is even, but in contrast to the situation for pseudo-ovals, when $q$ is odd, there are non-elementary examples of pseudo-ovoids. The standard example of an ovoid in $\mathrm{PG}\left(3, q^{n}\right)$ is an elliptic quadric $Q^{-}\left(3, q^{n}\right)$. By the famous result of Barlotti and Panella [1, 10], every ovoid of $\mathrm{PG}\left(3, q^{n}\right), q$ odd, is an elliptic quadric $Q^{-}\left(3, q^{n}\right)$, however, there is no classification of ovoids in $\mathrm{PG}\left(3, q^{n}\right)$ for $q$ even. For both even and odd order $q$, the classification of pseudo-ovoids is an open problem.

\section{Good eggs and Desarguesian spreads}

A (weak) egg $\mathcal{E}$ in $\mathrm{PG}(2 n+m-1, q), m>n$, is good at an element $E \in \mathcal{E}$ if every $(3 n-1)$-space containing $E$ and at least two other elements of $\mathcal{E}$, contains exactly $q^{n}+1$ 
elements of $\mathcal{E}$. A (weak) egg that has at least one good element is called a good (weak) egg. If $\mathcal{E}$ is good at $E$, then for any two elements $E_{1}, E_{2} \in \mathcal{E} \backslash\{E\}$ the $(3 n-1)$-space $\left\langle E, E_{1}, E_{2}\right\rangle$ intersects $\mathcal{E}$ in a pseudo-oval.

Lemma 8. Good weak eggs in $\mathrm{PG}(2 n+m-1, q)$ can only exist if $n$ is a divisor of $m$. Good eggs only exist in $\mathrm{PG}(4 n-1, q)$.

Proof. Consider a weak egg $\mathcal{E}$ of $\mathrm{PG}(2 n+m-1, q), m>n$, good at an element $E_{1} \in \mathcal{E}$. Consider a second element $E_{2} \in \mathcal{E} \backslash\left\{E_{1}\right\}$. For every element $E \in \mathcal{E} \backslash\left\{E_{1}, E_{2}\right\}$, the $(3 n-1)$-space $\left\langle E, E_{1}, E_{2}\right\rangle$ intersects $\mathcal{E}$ in a pseudo-oval. By considering the elements of $\mathcal{E} \backslash\left\{E_{1}, E_{2}\right\}$, we find a set $\mathcal{T}$ of $(3 n-1)$-spaces containing $\left\langle E_{1}, E_{2}\right\rangle$, such that each space of $\mathcal{T}$ intersects $\mathcal{E}$ in a pseudo-oval. Every two spaces in $\mathcal{T}$ meet exactly in $\left\langle E_{1}, E_{2}\right\rangle$ and $\mathcal{E}$ is the union of the pseudo-ovals $\{T \cap \mathcal{E} \mid T \in \mathcal{T}\}$. The set $\mathcal{T}$ contains $\frac{q^{m}-1}{q^{n}-1}(3 n-1)$-spaces; as $q^{n}-1$ has to be a divisor of $q^{m}-1$, it follows that $n$ is a divisor of $m$.

Suppose $\mathcal{E}$ is an egg. For $q$ even, by Theorem 4 , eggs only exist in $\mathrm{PG}(4 n-1, q)$ (or $\mathrm{PG}(3 n-1, q))$. Consider now a good egg of $\mathrm{PG}(2 n+m-1, q), q$ odd, where $m$ is a multiple of $n$. By Theorem $4, m=\frac{a+1}{a} n$, for some odd integer $a$, so we conclude that $m=2 n$.

We will show that the good elements of an egg are exactly those inducing a partial spread which is extendable to a Desarguesian spread. Part $(i)$ of the following theorem, for $\mathcal{E}$ an egg, is mentioned in [16, Remark 5.1.7].

\section{Theorem 9.}

(i) If a weak egg $\mathcal{E}$ in $\mathrm{PG}(2 n+m-1, q)$ is good at an element $E$, then $E$ induces a partial spread which extends to a Desarguesian spread.

(ii) Let $\mathcal{E}$ be a weak egg in $\mathrm{PG}(2 n+m-1, q)$ for $q$ odd and an egg in $\mathrm{PG}(2 n+m-1, q)$ for $q$ even. If an element $E \in \mathcal{E}$ induces a partial spread which extends to a Desarguesian spread, then $\mathcal{E}$ is good at $E$.

Proof. (i) Suppose $\mathcal{E}$ is a weak egg which is good at $E$. Consider the partial spread $\mathcal{S}$ of $\mathrm{PG}(n+m-1, q)$ of size $q^{m}$ induced by $E$. Because $\mathcal{E}$ is good at $E$, any two elements of $\mathcal{S}$ span a $(2 n-1)$-space which contains a partial spread of $q^{n}$ elements of $\mathcal{S}$. This partial spread has deficiency 1 , so extends uniquely to a spread by one $(n-1)$-space (by [3]).

Consider three elements $S_{1}, S_{2}, S_{3} \in \mathcal{S}$ not lying in the same $(2 n-1)$-space, hence spanning a $(3 n-1)$-space $\pi$. There are $q^{n}$ elements of $\mathcal{S}$ contained in $\left\langle S_{2}, S_{3}\right\rangle$. For every element $R$ of $\mathcal{S} \cap\left\langle S_{2}, S_{3}\right\rangle$, the $(2 n-1)$-space $\left\langle S_{1}, R\right\rangle$ contains $q^{n}$ elements of $\mathcal{S}$. Hence, there are $q^{n}(2 n-1)$-spaces of $\pi$ containing $S_{1}$ and $q^{n}-1$ other elements of $\mathcal{S}$. Similarly, there are $q^{n}(2 n-1)$-spaces of $\pi$ containing $S_{2}$ and $q^{n}-1$ other elements of $\mathcal{S}$. Since $\pi$ has dimension $3 n-1$, two such distinct $(2 n-1)$-spaces, one containing $S_{1}$ and the other containing $S_{2}$, intersect in at least an $(n-1)$-space, hence, in exactly an $(n-1)$-space. This space is either an element of $\mathcal{S}$ or the $(n-1)$-space which extends both of them to a spread. It follows that there are $q^{2 n}$ elements of $\mathcal{S}$ contained in $\pi$ and if an element of $\mathcal{S}$ 
intersects $\pi$, then it is contained in $\pi$. Hence, if $\left\langle S_{2}, S_{3}\right\rangle$ meets a $(2 n-1)$-space spanned by $S_{1}$ and an other element of $\mathcal{S}$, then they meet in an $(n-1)$-space.

As $S_{1}, S_{2}, S_{3}$ were chosen randomly, it follows in general that if two distinct $(2 n-1)$ spaces spanned by elements of $\mathcal{S}$ intersect, then they meet in an $(n-1)$-space. They meet either in an $(n-1)$-space of $\mathcal{S}$ or in the $(n-1)$-space which extends the partial spreads of both $(2 n-1)$-spaces to a spread. We see that $\mathcal{S}$ can be uniquely extended to a spread which is normal, thus Desarguesian.

(ii) Now let $\mathcal{E}$ be an egg if $q$ is even and a weak egg if $q$ is odd. Suppose $E$ induces a partial spread $\mathcal{S}$ of size $q^{m}$ which extends to a Desarguesian $(n-1)$-spread $\mathcal{D}$ of $\mathrm{PG}(n+$ $m-1, q)$, hence $m=k n$ for some $k>1$. There are $\frac{q^{m}-1}{q^{n}-1}$ elements of $\mathcal{D}$ not contained in $\mathcal{S}$.

When $\mathcal{E}$ is an egg, the elements of $\mathcal{D} \backslash \mathcal{S}$ span a $(m-1)$-space, corresponding to $T_{E}$. Hence, any $(2 n-1)$-space spanned by two elements of $\mathcal{S}$ contains $q^{n}$ elements of $\mathcal{S}$ and one element $\mathcal{D} \backslash \mathcal{S}$. So, $\mathcal{E}$ is good at $E$.

Suppose $\mathcal{E}$ is a weak egg, with $q$ odd. As $q$ is odd, no $(3 n-1)$-space intersects $\mathcal{E}$ in a pseudo-hyperoval. Hence, any $(3 n-1)$-space containing $E$ intersects $\mathcal{E}$ in at most $q^{n}+1$ elements, so any $(2 n-1)$-space spanned by two elements of $\mathcal{S}$ can contain at most $q^{n}$ elements of $\mathcal{S}$. Hence, any such space must contain at least one element of $\mathcal{D} \backslash \mathcal{S}$. By field reduction, the elements of the Desarguesian spread $\mathcal{D}$ of $\mathrm{PG}(n+m-1, q)$ are in one-toone correspondance with the points of $\operatorname{PG}\left(\frac{m}{n}, q^{n}\right)$. Any $(2 n-1)$-space spanned by two elements of $\mathcal{D}$ must contain at least one element of $\mathcal{D} \backslash \mathcal{S}$. Hence, the points corresponding to $\mathcal{D} \backslash \mathcal{S}$ form a line-blocking set of $\operatorname{PG}\left(\frac{m}{n}, q^{n}\right)$. Since $|\mathcal{D} \backslash \mathcal{S}|=\frac{q^{m}-1}{q^{n}-1}$, from [4] it follows that the points corresponding to $\mathcal{D} \backslash \mathcal{S}$ are the points of a $\left(\frac{m}{n}-1\right)$-space, hence the elements of $\mathcal{D} \backslash \mathcal{S}$ span a $(m-1)$-space. As before, it follows that $\mathcal{E}$ is good at $E$.

The following corollary, for $\mathcal{E}$ an egg, was also mentioned in [14, Theorem 4.3.4] in terms of translation generalised quadrangles.

Corollary 10. If a weak egg $\mathcal{E}, q$ odd, is good at an element $E$, then every pseudo-oval of $\mathcal{E}$ containing $E$ is a pseudo-conic.

Proof. Let $\Pi$ be a $(n+m-1)$-space disjoint from $E$. By Theorem 9, the partial spread $\mathcal{E} / E$ in $\Pi$ extends to a Desarguesian spread. Consider a pseudo-oval $\mathcal{O}$ of $\mathcal{E}$ containing $E$. The $q^{n}$ elements of $\mathcal{O} / E$ are contained in $\mathcal{E} / E$ and thus extend to a Desarguesian spread of the $(2 n-1)$-space $\langle\mathcal{O}\rangle \cap \Pi$.

The element $E$ of the pseudo-oval $\mathcal{O}$ induces a partial spread $\mathcal{O} / E$ which extends to a Desarguesian spread, hence, by Theorem 5 , the statement follows.

\section{A characterisation of good eggs}

\subsection{Eggs with two good elements}

An elementary pseudo-ovoid that arises from applying field reduction to an elliptic quadric is called classical. We recall the following theorem from [16]. 
Theorem 11. [16, Theorem 5.1.12]

If $q$ is odd and an egg $\mathcal{E}$ in $\mathrm{PG}(4 n-1, q)$ has at least two good elements, then $\mathcal{E}$ is classical. If $q$ is even and an egg $\mathcal{E}$ in $\mathrm{PG}(4 n-1, q)$ has at least four good elements, not contained in a common pseudo-oval on $\mathcal{E}$, then $\mathcal{E}$ is elementary.

It was open problem whether, for $q$ even, being good at two elements is sufficient to be elementary, this was posed as Problem A.5.6 in [16]. We will give an affirmative answer to this question in a more general setting, namely in terms of pseudo-caps. We first need two lemma's concerning Desarguesian spreads.

Lemma 12. [13, Corollary 1.8] Consider two Desarguesian $(n-1)$-spreads $\mathcal{S}_{1}$ and $\mathcal{S}_{2}$ in $\mathrm{PG}(2 n-1, q), q>2$. If $\mathcal{S}_{1}$ and $\mathcal{S}_{2}$ have at least 3 elements in common, then they share exactly $q^{t}+1$ elements for some $t \mid n$.

The following lemma is a generalisation of [13, Lemma 1.4] and the proof is analogous. We introduce some necessary definitions and notations.

A regulus $\mathcal{R}$ in $\operatorname{PG}(2 n-1, q)$ is a set of $q+1$ mutually disjoint $(n-1)$-spaces having the property that if a line meets 3 elements of $\mathcal{R}$, then it meets all elements of $\mathcal{R}$. Let us denote the unique regulus through 3 mutually disjoint $(n-1)$-spaces $A, B$ and $C$ in $\mathrm{PG}(2 n-1, q)$ by $\mathcal{R}(A, B, C)$. Every Desarguesian spread $\mathcal{D}$ has the property that for 3 elements $A, B, C$ in $\mathcal{D}$, the elements of $\mathcal{R}(A, B, C)$ are also contained in $\mathcal{D}$, i.e. $\mathcal{D}$ is regular (see also [5]).

We will use the following notation for points of a projective space $\mathrm{PG}\left(r-1, q^{n}\right)$. A point $P$ of $\mathrm{PG}\left(r-1, q^{n}\right)$ defined by a vector $\left(x_{1}, x_{2}, \ldots, x_{r}\right) \in\left(\mathbb{F}_{q^{n}}\right)^{r}$ is denoted by

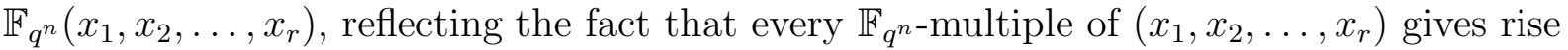
to the point $P$. We can identify the vector space $\mathbb{F}_{q^{n r}}$ with $\left(\mathbb{F}_{q^{n}}\right)^{r}$, and hence write every point of $\operatorname{PG}(r n-1, q)$ as $\mathbb{F}_{q}\left(x_{1}, \ldots, x_{r}\right)$ with $x_{i} \in \mathbb{F}_{q^{n}}$. In this way, when applying field reduction, a point $\mathbb{F}_{q^{n}}\left(x_{1}, \ldots, x_{r}\right)$ in $\mathrm{PG}\left(r-1, q^{n}\right)$ corresponds to the $(n-1)$-space $\mathbb{F}_{q^{n}}\left(x_{1}, \ldots, x_{r}\right)=\left\{\mathbb{F}_{q}\left(\alpha x_{1}, \ldots, \alpha x_{r}\right) \mid \alpha \in \mathbb{F}_{q^{n}}\right\}$ of $\operatorname{PG}(r n-1, q)$.

Lemma 13. Let $\mathcal{D}_{1}$ be a Desarguesian $(n-1)$-spread in a $(k n-1)$-dimensional subspace $\Pi$ of $\mathrm{PG}((k+1) n-1, q)$, let $\mu$ be an element of $\mathcal{D}_{1}$ and let $E_{1}$ and $E_{2}$ be mutually disjoint $(n-1)$-spaces such that $\left\langle E_{1}, E_{2}\right\rangle$ meets $\Pi$ exactly in the space $\mu$. Then there exists a unique Desarguesian $(n-1)$-spread of $\mathrm{PG}((k+1) n-1, q)$ containing $E_{1}, E_{2}$ and all elements of $\mathcal{D}_{1}$.

Proof. Since $\mathcal{D}_{1}$ is a Desarguesian spread in $\Pi$, we can choose coordinates for $\Pi$ such that $\mathcal{D}_{1}=\left\{\mathbb{F}_{q^{n}}\left(x_{1}, x_{2}, \ldots, x_{k}\right) \mid x_{i} \in \mathbb{F}_{q^{n}}\right\}$ and $\mu=\mathbb{F}_{q^{n}}(0, \ldots, 0,1)$. We embed $\Pi$ in $\operatorname{PG}((k+$ 1) $n-1, q)$ by mapping a point $\mathbb{F}_{q}\left(x_{1}, \ldots, x_{k}\right), x_{i} \in \mathbb{F}_{q^{n}}$, of $\Pi$ onto $\mathbb{F}_{q}\left(x_{1}, \ldots, x_{k}, 0\right)$. Let $\ell_{P}$ denote the unique transversal line through a point $P$ of $\mu$ to the regulus $\mathcal{R}\left(\mu, E_{1}, E_{2}\right)$.

We can still choose coordinates for $n+1$ points in general position in $\mathrm{PG}((k+1) n-$ $1, q) \backslash \Pi$. We will choose these $n+1$ points such that $n$ of them belong to $E_{1}$ and one of them belongs to $E_{2}$. Consider a set $\left\{y_{i} \mid i=1, \ldots, n\right\}$ forming a basis of $\mathbb{F}_{q^{n}}$ over $\mathbb{F}_{q}$. We may assume that the line $\ell_{P_{i}}$ through $P_{i}=\mathbb{F}_{q}\left(0, \ldots, 0, y_{i}, 0\right) \in \mu$ meets $E_{1}$ in the point $\mathbb{F}_{q}\left(0, \ldots, 0,0, y_{i}\right)$. It follows that $E_{1}=\mathbb{F}_{q^{n}}(0, \ldots, 0,0,1)$. Moreover, we may assume 
that $\ell_{Q}$ with $Q=\mathbb{F}_{q}\left(0, \ldots, 0,0, \sum_{i=1}^{n} y_{i}, 0\right) \in \mu$ meets $E_{2}$ in $\mathbb{F}_{q}\left(0, \ldots, 0, \sum_{i=1}^{n} y_{i}, \sum_{i=1}^{n} y_{i}\right)$. Since $\mathbb{F}_{q}\left(0, \ldots, 0 \sum_{i=1}^{n} y_{i}, \sum_{i=1}^{n} y_{i}\right)$ has to be in the space spanned by the intersection points $R_{i}=\ell_{P_{i}} \cap E_{2}$, it follows that $R_{i}=\mathbb{F}_{q}\left(0, \ldots, 0, y_{i}, y_{i}\right)$ and consequently, that $E_{2}=\mathbb{F}_{q^{n}}(0, \ldots, 0,1,1)$.

It is clear that the Desarguesian spread $\mathcal{D}=\left\{\mathbb{F}_{q^{n}}\left(x_{1}, \ldots, x_{k+1}\right) \mid x_{i} \in \mathbb{F}_{q^{n}}\right\}$ contains the spread $\mathcal{D}_{1}$ and the $(n-1)$-spaces $E_{1}$ and $E_{2}$. Moreover, since every element of $\mathcal{D}$, not in $\left\langle E_{1}, E_{2}\right\rangle$, is obtained as the intersection of $\left\langle E_{1}, X\right\rangle \cap\left\langle E_{2}, Y\right\rangle$, where $X, Y \in \mathcal{D}_{1}$, it is clear that $\mathcal{D}$ is the unique Desarguesian spread satisfying our hypothesis.

Lemma 14. Consider a pseudo-cap $\mathcal{E}$ of $\mathrm{PG}(4 n-1, q)$ containing an element $E$ that induces a partial spread which extends to a Desarguesian spread. If $\Pi$ is a $(3 n-1)$-space spanned by $E$ and two other elements of $\mathcal{E}$, then every element of $\mathcal{E}$ is either disjoint from $\Pi$ or contained in $\Pi$.

Proof. Let $\Sigma$ be a $(3 n-1)$-space skew from $E$ and consider the induced partial spread $\mathcal{E} / E$ in $\Sigma$. If $F$ is an element of $\mathcal{E}$ which meets $\Pi$, then the projection $F / E$ of $F$ from $E$ onto $\Sigma$ is an element of $\mathcal{E} / E$ which meets the space $\Pi / E$. By assumption, the space $\Pi / E$ is spanned by spread elements of a partial spread extending to a Desarguesian spread. Hence, since a Desarguesian spread is normal, $F / E$ is contained in $\Pi / E$. It follows that since $\Pi$ contains $E$, the element $F$ is contained in $\Pi$.

Theorem 15. Consider a pseudo-cap $\mathcal{E}$ in $\mathrm{PG}(4 n-1, q), q>2$, with $|\mathcal{E}|>q^{n+k}+q^{n}$ $q^{k}+1, q$ odd, and $|\mathcal{E}|>q^{n+k}+q^{n}+2$, q even, where $k$ is the largest divisor of $n$ with $k \neq n$. The pseudo-cap $\mathcal{E}$ is elementary if and only if two of its elements induce a partial spread which extends to a Desarguesian spread.

Proof. If $\mathcal{E}$ is elementary, then the elements of $\mathcal{E}$ are contained in a Desarguesian spread of $\mathrm{PG}(4 n-1, q)$, so every element of $\mathcal{E}$ induces a partial spread which extends to a Desarguesian spread.

Now suppose that $\mathcal{E}$ contains two distinct elements $E_{1}, E_{2}$ that induce a partial spread which extends to a Desarguesian spread. Since $|\mathcal{E}|>q^{n}+2$, using Lemma 14, we can find two elements $E_{3}, E_{4} \in \mathcal{E}$ such that $\left\langle E_{1}, E_{2}, E_{3}, E_{4}\right\rangle$ spans $\mathrm{PG}(4 n-1, q)$.

The partial spread induced by $E_{1}$ in the space $\left\langle E_{2}, E_{3}, E_{4}\right\rangle$ can be extended to a Desarguesian spread $\mathcal{D}_{1}$. Analogously, the partial spread induced by $E_{2}$ in the space $\left\langle E_{1}, E_{3}, E_{4}\right\rangle$ can be extended to a Desarguesian spread $\mathcal{D}_{2}$. Since $E_{3}$ and $E_{4}$ are elements of the spreads $\mathcal{D}_{1}$ and $\mathcal{D}_{2}$, the Desarguesian spreads $\mathcal{D}_{1}$ and $\mathcal{D}_{2}$ intersect the $(2 n-1)$-space $\left\langle E_{3}, E_{4}\right\rangle$ each in a Desarguesian spread, say $\mathcal{S}_{1}$ and $\mathcal{S}_{2}$ respectively.

Take an element $E \in \mathcal{E} \backslash\left\{E_{1}, E_{2}\right\}$ and consider the $(3 n-1)$-subspace $\left\langle E_{1}, E_{2}, E\right\rangle$. From Lemma 14 it follows that any element of $\mathcal{E}$ is either contained in or disjoint from $\left\langle E_{1}, E_{2}, E\right\rangle$. By considering the elements of $\mathcal{E} \backslash\left\{E_{1}, E_{2}\right\}$, we find a set $\mathcal{T}$ of $(3 n-1)$-spaces containing $\left\langle E_{1}, E_{2}\right\rangle$, such that each space of $\mathcal{T}$ intersects $\mathcal{E}$ in a pseudo-arc. Every two spaces in $\mathcal{T}$ meet exactly in $\left\langle E_{1}, E_{2}\right\rangle$ and $\mathcal{E}$ is the union of the pseudo-arcs $\{T \cap \mathcal{E} \mid T \in \mathcal{T}\}$. The set $\mathcal{T}$ intersects $\left\langle E_{3}, E_{4}\right\rangle$ in a partial $(n-1)$-spread $\mathcal{P}$.

Let $P$ be an element of $\mathcal{P}$, then $\left\langle P, E_{1}, E_{2}\right\rangle$ is a $(3 n-1)$-space containing at least one element $E$ of $\mathcal{E} \backslash\left\{E_{1}, E_{2}\right\}$. The projection $E^{\prime}$ of $E$ from $E_{1}$ onto $\left\langle E_{2}, E_{3}, E_{4}\right\rangle$ is contained 
in $\mathcal{D}_{1}$. We obtain that $P=\left\langle E_{2}, E^{\prime}\right\rangle \cap\left\langle E_{3}, E_{4}\right\rangle$, and since the elements $E_{2}, E^{\prime}, E_{3}, E_{4}$ are contained in $\mathcal{D}_{1}$, this implies that $P$ is contained in $\mathcal{D}_{1}$. Moreover, since $P \subset\left\langle E_{3}, E_{4}\right\rangle$, the element $P$ is contained in $\mathcal{S}_{1}$. Similarly, we obtain that $P$ is contained in $\mathcal{S}_{2}$ and we conclude that every element of $\mathcal{P}$ must be contained in both $\mathcal{S}_{1}$ and $\mathcal{S}_{2}$.

Suppose that $k$ is the largest divisor of $n$ with $k \neq n$. The pseudo-cap $\mathcal{E}$ has size $|\mathcal{E}|>\left(q^{n}-\epsilon\right)\left(q^{k}+1\right)+2$ and every $(3 n-1)$-space of $\mathcal{T}$ contains at most $q^{n}-\epsilon$ elements different from $E_{1}, E_{2}$, where $\epsilon=1$ for $q$ odd and $\epsilon=0$ for $q$ even. By the pigeonhole principle, it follows that $|\mathcal{P}| \geqslant q^{k}+2$. Hence, the Desarguesian spreads $\mathcal{S}_{1}$ and $\mathcal{S}_{2}$ have at least $q^{k}+2$ elements in common, where $k$ is the largest divisor of $n$ with $k \neq n$. As $q>2$, by Lemma 12, we find that $\mathcal{S}_{1}=\mathcal{S}_{2}$.

By Theorem 13, consider the unique Desarguesian spread $\mathcal{D}$ of $\mathrm{PG}(4 n-1, q)$ containing all elements of $\mathcal{D}_{1}$ and two distinct elements of $\mathcal{D}_{2} \backslash \mathcal{D}_{1}$. It is clear that, since $\mathcal{S}_{1}=\mathcal{S}_{2}$, the spread $\mathcal{D}$ contains all elements of $\mathcal{D}_{2}$.

Every element of $\mathcal{E}$, not in $\mathcal{D}_{1} \cup \mathcal{D}_{2}$, arises as the intersection $\left\langle E_{1}, X\right\rangle \cap\left\langle E_{2}, Y\right\rangle$ for some $X \in \mathcal{D}_{1} \subset \mathcal{D}$ and $Y \in \mathcal{D}_{2} \subset \mathcal{D}$, hence, since a Desarguesian spread is normal, every element of $\mathcal{E}$ belongs to $\mathcal{D}$. It follows that $\mathcal{E}$ is elementary.

We obtain the following corollary which improves [16, Theorem 5.1.12].

Corollary 16. A weak egg in $\mathrm{PG}(4 n-1, q)$ which is good at two distinct elements is elementary.

Proof. A weak egg is a pseudo-cap of size $q^{2 n}+1$ in $\mathrm{PG}(4 n-1, q)$. By Theorem 9 , if the weak egg is good at two elements, these elements induce a partial spread which extends to a Desarguesian spread. We can repeat the proof of Theorem 15. Now the partial spread $\mathcal{P}$ has size $q^{n}+1$, so the conclusion $\mathcal{S}_{1}=\mathcal{S}_{2}$ follows immediately. We do not require Lemma 12, hence the restriction $q>2$ can be dropped.

\subsection{A corollary in terms of translation generalised quadrangles}

A generalised quadrangle of order $(s, t), s, t>1$, is an incidence structure of points and lines satisfying the following axioms:

- every line has exactly $s+1$ points,

- through every point, there are exactly $t+1$ lines,

- if $P$ is a point, not on the line $L$, then there is exactly one line through $P$ which meets $L$ non-trivially.

From every egg $\mathcal{E}$ in $\Sigma_{\infty}=\operatorname{PG}(2 n+m-1, q)$ we can construct a generalised quadrangle $(\mathcal{P}, \mathcal{L})$ as follows. Embed $\Sigma_{\infty}$ as a hyperplane at infinity of $\operatorname{PG}(2 n+m, q)$.

$\mathcal{P}: \quad(i)$ affine points of $\operatorname{PG}(2 n+m, q)$, i.e. the points not lying in $\Sigma_{\infty}$,

(ii) the $(n+m)$-spaces meeting $\Sigma_{\infty}$ in $T_{E}$ for some $E \in \mathcal{E}$,

(iii) the symbol $(\infty)$. 
$\mathcal{L}: \quad(a)$ the $n$-spaces meeting $\Sigma_{\infty}$ in an element of $\mathcal{E}$,

(b) the elements of $\mathcal{E}$.

Incidence is defined as follows.

- A point of type $(i)$ is incident with the lines of type $(a)$ through it.

- A point of type $(i i)$ is incident with the lines of type $(a)$ it contains and the line of type $(b)$ it contains.

- The point $(\infty)$ is incident with all lines of type $(b)$.

The obtained generalised quadrangle is denoted as $T(\mathcal{E})$ and is called a translation generalised quadrangle (TGQ) with base point $(\infty)$. In [11, Theorem 8.7.1] it is proven that every TGQ of order $\left(q^{n}, q^{m}\right)$, where $\mathbb{F}_{q}$ is a subfield of its kernel, is isomorphic to a $T(\mathcal{E})$ for some egg $\mathcal{E}$ of $\mathrm{PG}(2 n+m-1, q)$.

When $n=m=1$, then $\mathcal{O}$ is an oval of $\operatorname{PG}(2, q)$ and the construction above gives the well-known construction of $T_{2}(\mathcal{O})$. When $n=1$ and $m=2$, then $\mathcal{O}$ is an ovoid of $\operatorname{PG}(3, q)$ and the construction above is the construction of Tits of $T_{3}(\mathcal{O})$ (see [16]).

Lemma 17. Let $T=T(\mathcal{E})$ be a $T G Q$ of order $\left(q^{n}, q^{2 n}\right)$ with base point $(\infty)$. Let $m_{1}, m_{2}, m_{3}$ be three distinct lines through $(\infty)$, and let $E_{1}, E_{2}, E_{3}$ denote the elements of $\mathcal{E}$ corresponding to $m_{1}, m_{2}, m_{3}$ respectively. Then there is a subquadrangle of order $q^{n}$ through $m_{1}, m_{2}, m_{3}$ if and only if the $(3 n-1)$-dimensional space $\left\langle E_{1}, E_{2}, E_{3}\right\rangle$ contains exactly $q^{n}+1$ elements of $\mathcal{E}$.

Proof. Suppose that the $(3 n-1)$-space $\Sigma=\left\langle E_{1}, E_{2}, E_{3}\right\rangle$ contains a set $\mathcal{O}$ of exactly $q^{n}+1$ elements of $\mathcal{E}$, then it is clear that $T(\mathcal{E})$ defines the incidence structure $T(\mathcal{O})$ in a $3 n$-space through $\Sigma$. The structure $T(\mathcal{O})$ is a generalised quadrangle of order $q^{n}$, forming a subquadrangle of $T(\mathcal{E})$ and containing the lines $m_{1}, m_{2}, m_{3}$.

On the other hand, suppose that there is a subquadrangle $T^{\prime}$ of order $q^{n}$ containing $m_{1}, m_{2}, m_{3}$, where the lines $m_{1}, m_{2}, m_{3}$ are incident with $(\infty)$. This implies that the point $(\infty)$ is in $T^{\prime}$, and since $(\infty)$ lies only on lines of type $(b)$ (i.e. the lines corresponding to elements of $\mathcal{E}$ ), we know that $T^{\prime}$ contains exactly $q^{n}+1$ lines of type $(b)$, among which the lines $m_{1}, m_{2}$ and $m_{3}$. Let $\left\{E_{1}, \ldots, E_{q^{n}+1}\right\}$ be the egg elements corresponding to these lines. This means that there are $\left(q^{n}+1\right) q^{2 n}$ lines in $T^{\prime}$ of type $(a)$, containing in total $\left(q^{n}+1\right) q^{2 n}\left(q^{n}\right) /\left(q^{n}+1\right)=q^{3 n}$ points of type $(i)$ (i.e. affine points).

Each $(n-1)$-space $E_{j}$ is contained in $q^{2 n} n$-spaces corresponding to a line of type $(a)$ of $T^{\prime}$ and every affine point is contained in exactly one $n$-space containing $E_{j}$. Let $P_{j}$ be a point of the space $E_{j}$, then we see that the $q^{3 n}$ affine points of $T^{\prime}$ lie on $q^{2 n}$ lines through $P_{j}$. As this holds for every $j \in\left\{1, \ldots, q^{n}+1\right\}$, it is clear that the $q^{3 n}$ affine points of $T^{\prime}$ are contained in a $3 n$-space. This in turn implies that the elements $\left\{E_{1}, \ldots, E_{q^{n}+1}\right\}$ are contained in a $(3 n-1)$-space, namely $\left\langle E_{1}, E_{2}, E_{3}\right\rangle$. Hence, this space contains at least $q^{n}+1$ elements of $\mathcal{E}$. Since $\mathcal{E}$ is an egg, it is not possible that a $(3 n-1)$-space contains more than $q^{n}+1$ elements of $\mathcal{E}$, which concludes the proof. 
Lemma 18. Let $T=T(\mathcal{E})$ be a $T G Q$ of order $\left(q^{n}, q^{2 n}\right)$ with base point $(\infty)$. Let $\ell$ be a line through $(\infty)$ and $E_{\ell}$ the element of $\mathcal{E}$ corresponding to $\ell$. The egg $\mathcal{E}$ is good at $E_{\ell}$ if and only if for every two distinct lines $m_{1}, m_{2}$ through $(\infty)$, where $m_{1}, m_{2} \neq \ell$, there is a subquadrangle of order $q^{n}$ through $m_{1}, m_{2}, \ell$.

Proof. This follows immediately from Lemma 17 and the definition of a being good at an element.

We are now ready to state the promised characterisation of the translation generalised quadrangle $T_{3}(\mathcal{O})$.

Theorem 19. Let T be a TGQ of order $\left(q^{n}, q^{2 n}\right)$ with base point $(\infty)$. Suppose that $T$ contains two distinct lines $\ell_{i}, i=1,2$ such that for every two distinct lines $m_{1}, m_{2}$ through $(\infty)$, where $m_{1}, m_{2} \neq \ell_{i}, i=1,2$ there is a subquadrangle through $m_{1}, m_{2}, \ell_{i}, i=1,2$, then $T$ is isomorphic to $T_{3}(\mathcal{O})$, where $\mathcal{O}$ is an ovoid of $\mathrm{PG}\left(3, q^{n}\right)$.

\section{A geometric proof of a Theorem of Lavrauw}

In this section we obtain a second characterisation of good weak eggs. We need the following lemma stating that every good element of a weak egg has a tangent space.

Lemma 20. If a weak egg $\mathcal{E}$ in $\mathrm{PG}(2 n+m-1, q)$ is good at an element $E$, then there exists a unique $(n+m-1)$-space $T$, such that $T \cap \mathcal{E}=\{E\}$.

Proof. Consider a $(n+m-1)$-space $\Sigma$ disjoint from $E$. If $\mathcal{E}$ is good at $E$, the element $E$ induces a partial spread $\mathcal{S}=\mathcal{E} / E$ which extends to a Desarguesian spread $\mathcal{D}$ of $\Sigma$. By following the proof of Theorem 9, part (ii), for both $q$ odd and $q$ even, the elements of $\mathcal{D} \backslash \mathcal{S}$ span a $(m-1)$-space. It is clear that the $(n+m-1)$-space $T=\langle E, \mathcal{D} \backslash \mathcal{S}\rangle$ satisfies $T \cap \mathcal{E}=E$.

In [8] the authors proved that every egg of $\mathrm{PG}(7,2)$ arises from an elliptic quadric $Q^{-}(3,4)$ by field reduction. Hence, in the following characterisation, when $\mathcal{E}$ is an egg in $\mathrm{PG}(4 n-1, q)$, the condition $q^{n}>4$ is essentially not a restriction.

Theorem 21. Suppose $n>1, q^{n}>4$, consider $\mathcal{E}$ a weak egg in $\mathrm{PG}(4 n-1, q)$. Then $\mathcal{E}$ is elementary if and only if the following three properties hold:

- $\mathcal{E}$ is good at an element $E$,

- there exists a $(3 n-1)$-space, disjoint from $E$, with at least 5 elements $E_{1}, E_{2}, E_{3}$, $E_{4}, E_{5}$ of $\mathcal{E}$,

- all pseudo-ovals of $\mathcal{E}$ containing $\left\{E, E_{1}\right\},\left\{E, E_{2}\right\}$ or $\left\{E, E_{3}\right\}$ are elementary. 
Proof. Clearly, if an egg is elementary, the statement is valid.

For the converse, consider the $(3 n-1)$-space $\Pi$ containing 5 elements $E_{1}, E_{2}, E_{3}, E_{4}, E_{5}$ of $\mathcal{E}$, but not the element $E$. As $\mathcal{E}$ is good at $E$, the element $E$ induces a partial spread which extends to a Desarguesian $(n-1)$-spread $\mathcal{D}_{0}$ in $\Pi$, which contains $E_{i}, i=1, \ldots, 5$.

By Lemma 20, there exists a unique $(3 n-1)$-space $T$, such that $T \cap \mathcal{E}=\{E\}$. When $\mathcal{E}$ is an egg, this space corresponds to the tangent space $T_{E}$.

Consider the two $(n-1)$-spaces $F=\left\langle E_{1}, E_{5}\right\rangle \cap\left\langle E_{2}, E_{4}\right\rangle$ and $F^{\prime}=\left\langle E_{1}, E_{5}\right\rangle \cap\left\langle E_{3}, E_{4}\right\rangle$. Both $F$ and $F^{\prime}$ are contained in $\mathcal{D}_{0}$, but at most one of them can be contained in the $(2 n-1)$-space $\Pi \cap T$. Suppose $F$ is not contained in $T$ (note that this choice has no further impact as $E_{2}$ and $E_{3}$ play the same role). This implies that the $(2 n-1)$-space $\langle E, F\rangle$ contains an element $E_{6} \in \mathcal{E} \backslash\{E\}$. By Theorem 13, there exists a unique Desarguesian spread $\mathcal{D}$ containing $E, E_{6}$ and all elements of $\mathcal{D}_{0}$. We will prove that $\mathcal{E}$ is contained in $\mathcal{D}$.

The $(3 n-1)$-space $\left\langle E, E_{1}, E_{5}\right\rangle$ intersect $\mathcal{E}$ in a pseudo-oval $\mathcal{O}_{1}$, and the $(3 n-1)$-space $\left\langle E, E_{2}, E_{4}\right\rangle$ intersect $\mathcal{E}$ in a pseudo-oval $\mathcal{O}_{2}$. Clearly, $\mathcal{O}_{1}$ and $\mathcal{O}_{2}$ both contain $E_{6}$.

By assumption, $\mathcal{O}_{1}$ and $\mathcal{O}_{2}$ are elementary pseudo-ovals. The Desarguesian $(n-1)$ spread in $\left\langle E, E_{1}, E_{5}\right\rangle$ containing $\mathcal{O}_{1}$ contains $E, E_{6}$ and the $q^{n}+1$ elements of $\mathcal{D}_{0} \cap\left\langle E_{1}, E_{5}\right\rangle$. It follows that this Desarguesian spread is contained in $\mathcal{D}$, hence $\mathcal{O}_{1}$ is contained in $\mathcal{D}$. Analogously, the pseudo-oval $\mathcal{O}_{2}$ is also contained in $\mathcal{D}$.

There are $q^{n}-2$ pseudo-ovals $\mathcal{O}$ of $\mathcal{E}$, containing $\left\{E, E_{3}\right\}$, but not $E_{6}$, such that the $(3 n-1)$-space $\langle\mathcal{O}\rangle$ does not contain the $(n-1)$-space $T \cap\left\langle\mathcal{O}_{1}\right\rangle$, nor the $(n-1)$-space $T \cap\left\langle\mathcal{O}_{2}\right\rangle$. Take such an oval $\mathcal{O}$, then there is an element $E_{7}$ of $\mathcal{E} \backslash\{E\}$ contained in $\langle\mathcal{O}\rangle \cap\left\langle\mathcal{O}_{1}\right\rangle$, hence, $E_{7} \in \mathcal{O} \cap \mathcal{O}_{1}$. Likewise, there is an element $E_{8}$ of $\mathcal{E} \backslash\{E\}$ contained in $\mathcal{O} \cap \mathcal{O}_{2}$.

By assumption, $\mathcal{O}$ is elementary; let $\mathcal{S}_{\mathcal{O}}$ be the Desarguesian $(n-1)$-spread containing $\mathcal{O}$. As $E_{7}$ and $E_{8}$ are contained in $\mathcal{D}$, the Desarguesian spread $\mathcal{D}$ intersects $\left\langle E_{7}, E_{8}\right\rangle$ in a Desarguesian spread. Let $P$ be an element of $\mathcal{D} \cap\left\langle E_{7}, E_{8}\right\rangle$, not contained in $T$, then $\langle E, P\rangle$ meets $\Pi$ in an element of $\mathcal{D}$, and hence, $\langle E, P\rangle$ contains an element $P^{\prime}$ of $\mathcal{E} \backslash E$. As $\langle E, P\rangle$ is contained in $\langle\mathcal{O}\rangle, P^{\prime}$ is an element of $\mathcal{O}$, and hence also of $\mathcal{S}_{\mathcal{O}}$. Since $P^{\prime}, E, E_{7}, E_{8}$ are contained in $\mathcal{S}_{\mathcal{O}}$, the element $P=\left\langle E, P^{\prime}\right\rangle \cap\left\langle E_{7}, E_{8}\right\rangle$ is an element of $\mathcal{S}_{\mathcal{O}}$. This implies that $\mathcal{D} \cap\left\langle E_{7}, E_{8}\right\rangle$ and $\mathcal{S}_{\mathcal{O}}$ have at least $q^{n}$ elements in common, which implies in turn that they have all their elements in common. We conclude that $\mathcal{S}_{\mathcal{O}}$ contains $E, E_{3}$ and the $q^{n}+1$ elements of $\mathcal{D} \cap\left\langle E_{7}, E_{8}\right\rangle$, hence $\mathcal{S}_{\mathcal{O}}$ and thus all elements of $\mathcal{O}$ are contained in $\mathcal{D}$.

Now, consider an element $E_{9} \in \mathcal{E}$, not contained in $\mathcal{O}_{1}, \mathcal{O}_{2}$ or any of the previously considered $q^{n}-2$ pseudo-ovals $\mathcal{O}$. Look at the pseudo-oval $\mathcal{O}^{\prime}=\left\langle E, E_{1}, E_{9}\right\rangle \cap \mathcal{E}$ and the pseudo-oval $\mathcal{O}^{\prime \prime}=\left\langle E, E_{2}, E_{9}\right\rangle \cap \mathcal{E}$. At least one of them does not contain $E_{3}$. Suppose $\mathcal{O}^{\prime}$ does not contain $E_{3}$ (the proof goes analogously if $\mathcal{O}^{\prime \prime}$ does not contain $E_{3}$ ). For at most one of the $q^{n}-2$ pseudo-ovals $\mathcal{O}$ containing $\left\{E, E_{3}\right\}$ we have $\langle\mathcal{O}\rangle \cap\left\langle\mathcal{O}^{\prime \prime}\right\rangle \in T$. Hence, since $q^{n}-2 \geqslant 3$, we can find two distinct elementary pseudo-ovals containing $\left\{E, E_{3}\right\}$ that are contained in $\mathcal{D}$ and have an element $E_{10}$ and $E_{11}$ respectively in common with $\mathcal{O}^{\prime}$.

Let $\mathcal{S}_{\mathcal{O}^{\prime}}$ be the Desarguesian $(n-1)$-spread containing $\mathcal{O}^{\prime}$. As $E_{10}$ and $E_{11}$ are elements of $\mathcal{D}$ the same argument as before shows that all but one element of the Desarguesian 
spread $\mathcal{D} \cap\left\langle E_{10}, E_{11}\right\rangle$ can be written as the intersection of $\left\langle E, P^{\prime \prime}\right\rangle$ with $\left\langle E_{10}, E_{11}\right\rangle$ for some $P^{\prime \prime}$ in $\mathcal{O}^{\prime}$. It follows that $\mathcal{S}_{\mathcal{O}^{\prime}}$ contains $E, E_{1}$ and the $q^{n}+1$ elements of $\mathcal{D} \cap\left\langle E_{10}, E_{11}\right\rangle$, hence, that $\mathcal{S}_{\mathcal{O}^{\prime}}$ is contained in $\mathcal{D}$. In particular, the element $E_{9}$ is contained in $\mathcal{D}$, which implies that $\mathcal{E} \subset \mathcal{D}$ and so that $\mathcal{E}$ is elementary and more specifically, a field reduced ovoid.

When $\mathcal{E}$ is good at $E$ and $q$ is odd, by Corollary 10 all pseudo-ovals of $\mathcal{E}$ containing $E$ are pseudo-conics; we use this to obtain the following corollary. The same statement, where $\mathcal{E}$ is an egg, was proven in [7, Theorem 3.2] using coordinates. For $\mathcal{E}$ an egg, this was also shown in [16, Theorem 5.2.3] where a different proof was obtained independently, relying on a technical theorem concerning the $\mathbb{F}_{q^{n}}$-extension of the egg elements. We have now obtained a direct geometric proof.

Corollary 22. A weak egg $\mathcal{E}$ of $\mathrm{PG}(4 n-1, q), q$ odd, $n>1$, is classical if and only if it is good at an element $E$ and there exists a $(3 n-1)$-space, not containing $E$, with at least 5 elements of $\mathcal{E}$.

\section{Acknowledgements}

The authors wish to thank Simeon Ball for suggesting the study of eggs in terms of the induced (partial) spreads.

\section{References}

[1] A. Barlotti. Un'estensione del teorema di Segre-Kustaanheimo. Boll. Un. Mat. Ital. 3(10):498-506, 1955.

[2] A. Barlotti and J. Cofman. Finite Sperner spaces constructed from projective and affine spaces. Abh. Math. Sem. Univ. Hamburg 40:231-241, 1974.

[3] A. Beutelspacher. Blocking sets and partial spreads in finite projective spaces. Geom. Dedicata 9(4):425-449, 1980.

[4] R.C. Bose and R.C. Burton. A characterization of flat spaces in a finite geometry and the uniqueness of the Hamming and the MacDonald codes. J. Combin. Theory 1:96-104, 1966.

[5] R.H. Bruck and R.C. Bose. The construction of translation planes from projective spaces. J. Algebra 1:85-102, 1964.

[6] L.R.A. Casse, J.A. Thas and P.R. Wild. $\left(q^{n}+1\right)$-sets of PG $(3 n-1, q)$, generalized quadrangles and Laguerre planes. Simon Stevin 59(1):21-42, 1985.

[7] M. Lavrauw. Characterizations and properties of good eggs in PG(4n-1, $q), q$ odd. Discrete Math. 301:106-116, 2005.

[8] M. Lavrauw and T. Penttila. On eggs and translation generalised quadrangles. $J$. Combin. Theory Ser. A 96:303-315, 2001. 
[9] M. Lavrauw and G. Van de Voorde. Field reduction in finite geometry. Topics in finite fields. Contemp. Math., 632, Amer. Math. Soc., Providence, RI, 2010.

[10] G. Panella. Caratterizzazione delle quadriche di uno spazio (tridimensionale) lineare sopra un corpo finito. Boll. Un. Mat. Ital. 3(10):507-513, 1955.

[11] S.E. Payne and J.A. Thas. Finite generalized quadrangles. Research Notes in Mathematics, 110. Pitman (Advanced Publishing Program), Boston, MA, 1984. vi+312 pp. ISBN 0-273-08655-3.

[12] T. Penttila and G. Van de Voorde. Extending pseudo-arcs in odd characteristic. Finite Fields Appl. 22:101-113, 2013.

[13] S. Rottey and G. Van de Voorde. Pseudo-ovals in even characteristic and ovoidal Laguerre planes. J. Combin. Theory Ser. A 129:105-121, 2015.

[14] J.A. Thas. Generalized quadrangles of order $\left(s, s^{2}\right)$, I. J. Combin. Theory Ser. A 67:140-160, 1994.

[15] J.A. Thas. The m-dimensional projective space $S_{m}\left(M_{n}(G F(q))\right)$ over the total matrix algebra $M_{n}(G F(q))$ of the $n \times n$-matrices with elements in the Galois field $G F(q)$. Rend. Mat. 6(4):459-532, 1971.

[16] J.A. Thas, K. Thas and H. Van Maldeghem. Translation generalized quadrangles. Series in Pure Mathematics 26. World Scientific Publishing Co. Pte. Ltd., Hackensack, NJ, 2006. 